\title{
Big bang nucleosynthesis in the new cosmology
}

\author{
B.D. Fields ${ }^{\mathrm{a}}$ \\ Center for Theoretical Astrophysics, Departments of Astronomy and Physics, University of Illinois, Urbana, IL 61801, USA
}

Received: 30 June 2005 /

Published online: 13 February 2006 - (C) Società Italiana di Fisica / Springer-Verlag 2006

\begin{abstract}
Big bang nucleosynthesis (BBN) describes the production of the lightest elements in the first minutes of cosmic time. We review the physics of cosmological element production, and the observations of the primordial element abundances. The comparison between theory and observation has heretofore provided our earliest probe of the universe, and given the best measure of the cosmic baryon content. However, BBN has now taken a new role in cosmology, in light of new precision measurements of the cosmic microwave background (CMB). Recent CMB anisotropy data yield a wealth of cosmological parameters; in particular, the baryon-to-photon ratio $\eta=n_{B} / n_{\gamma}$ is measured to high precision. The confrontation between the BBN and CMB "baryometers" poses a new and stringent test of the standard cosmology; the status of this test is discussed. Moreover, it is now possible to recast the role of BBN by using the CMB to fix the baryon density and even some light element abundances. This strategy sharpens BBN into a more powerful probe of early universe physics, and of galactic nucleosynthesis processes. The impact of the CMB results on particle physics beyond the Standard Model, and on non-standard cosmology, are illustrated. Prospects for improvement of these bounds via additional astronomical observations and nuclear experiments are discussed, as is the lingering "lithium problem."
\end{abstract}

PACS. 98.80.Ft Origin, formation, and abundances of the elements $-26.35 .+\mathrm{c}$ Big Bang nucleosynthesis

\section{Introduction}

Big bang nucleosynthesis (BBN) offers the deepest reliable probe of the early universe, being based on well-understood Standard Model physics. Predictions of the abundances of the light elements, D, ${ }^{3} \mathrm{He},{ }^{4} \mathrm{He}$, and ${ }^{7} \mathrm{Li}$, synthesized at the end of the "first three minutes" are in good overall agreement with the primordial abundances inferred from observational data, thus validating the standard hot big bang cosmology (see [1]). This is particularly impressive given that these abundances span nine orders of magnitude - from ${ }^{4} \mathrm{He} / \mathrm{H} \sim 0.08$ down to ${ }^{7} \mathrm{Li} / \mathrm{H} \sim 10^{-10}$ (ratios by number). Thus BBN provides powerful constraints on possible deviations from the standard cosmology [2], and on new physics beyond the Standard Model [3].

We are presently entering an age of a "new cosmology," in which BBN plays a changing but crucial role. The basic world model of a hot big bang has been resoundingly confirmed by a wealth of new observations with unprecedented precision. The fundamental parameters of this world model, including the abundances of the matter and energy component of the cosmos, are now known to a few percent.

\footnotetext{
${ }^{a}$ e-mail: bdfields@uiuc.edu
}

The advent of "precision cosmology" is largely spurred by measurements of the cosmic microwave background radiation $(\mathrm{CMB})$. The $\mathrm{CMB}$ and $\mathrm{BBN}$ are intimately connected. This link traces back to the work of Gamow, Alpher, and Herman [4,5], who determined the thermodynamic conditions needed for nucleosynthesis in the early universe, and used this to extrapolate a present radiation temperature of order $T \sim 5 \mathrm{~K}$. This historic BBN-CMB connection has recently been deepened with the advent of high-precision measurements of the CMB anisotropy [6]. The measurements have led to determinations of cosmological parameters with an unprecedented accuracy. These include the baryon density, which is the sole parameter in standard BBN.

With the cosmic baryon density measured independently via $\mathrm{BBN}$ and the $\mathrm{CMB}$, several new analyses are possible. 1) First and foremost, one can pit BBN against the CMB, asking whether the results of the independent BBN and CMB "baryometers" agree. This comparison marks a fundamental and non-trivial test of the hot big bang. We will see, in fact, that cosmology passes this test, but then other strategies are possible when one combines $\mathrm{BBN}$ and the $\mathrm{CMB}$, taking advantage of the precision of the CMB baryon density determination. 2) For standard BBN, a CMB-based baryon density fixes all parameters. Then BBN simply makes definite predictions for the 
abundances of the light elements which can then be contrasted with their observational determinations [7]. The results shed new light on the nucleosynthesis of light elements after the big bang.

Finally, 3) one can relax the assumptions of standard BBN (e.g., an early universe populated only by known Standard Model particles and interactions). With the baryon density fixed by the CMB, for the first time, all light elements are available to probe early universe physics. This is perhaps the most exciting new possibility for BBN, and highlights the other aspect of the "new cosmology," namely that our view of the constituents, dynamics, and history of the cosmos has drastically changed of late. The universe appears to be accelerating today, due to a dark energy whose nature is unknown, but may provide clues to such far-reaching realms as quantum gravity. How and whether the dark energy is connected with another cosmic period of acceleration - inflation - remains to be clearly elucidated. Also, the need for and abundance of dark matter is ever more firmly established, but its nature and any connection with dark energy (or baryons!) is unclear. All of these open questions almost surely will find their answers in the early universe. And in this context it is crucial to appreciate that BBN provides our earliest reliable probe.

Thus the new cosmology presents both challenges and opportunities for BBN in general and for nuclear physics in particular. These will be the focus of this review, which draws heavily from [8] which adopts a very similar approach. For other recent reviews see [1].

\section{Big bang nucleosynthesis theory}

The theory of BBN consists of following the microphysics of weak and nuclear reactions in the cosmological context of an expanding, cooling universe. In fact, the essential aspects of BBN can be understood in terms of the competition between the cosmic expansion rate and particle reaction rates. The expansion rate $H=\dot{a} / a$, with $a(t)$ the cosmic scale factor, is given by the usual Friedmann equation:

$$
H^{2}=\frac{8 \pi G}{3} \rho,
$$

where we have dropped the curvature term and cosmological constant as both are negligible in the early universe compared with the energy density. In the early universe, the energy density was dominated by relativistic species ("radiation")

$$
\rho_{\text {rad }}=\frac{\pi^{2}}{30}\left(2+\frac{7}{2}+\frac{7}{4} N_{\nu}\right) T^{4} \equiv \frac{\pi^{2}}{30} g_{*} T^{4},
$$

which consists of photons, electrons and positrons, and $N_{\nu}$ neutrino flavors (at higher temperatures, other particle degrees of freedom should be included as well). Thus time and temperature scale as $t \sim 1 / H \sim 1 / T^{2}$, and in standard $\mathrm{BBN}$ (i.e., with $N_{\nu}=3$ ), we roughly have $t / 1 \mathrm{~s} \simeq\left(2.4 / g_{*}\right)(1 \mathrm{MeV} / T)^{2}$.
The synthesis of the light elements is sensitive to physical conditions in the early radiation-dominated era at temperatures $T \lesssim 1 \mathrm{MeV}$, corresponding to an age $t \gtrsim 1 \mathrm{~s}$. At these and higher temperatures, weak interactions rates $\Gamma_{\text {weak }} \gg H$ were rapid compared to the expansion rate, and thus the weak interactions were in thermal equilibrium. In particular, the processes

$$
\begin{aligned}
n+e^{+} & \leftrightarrow p+\bar{\nu}_{e}, \\
n+\nu_{e} & \leftrightarrow p+e^{-}, \\
n & \leftrightarrow p+e^{-}+\bar{\nu}_{e},
\end{aligned}
$$

fix the ratio of the neutron and proton number densities to be $n / p=e^{-Q / T}$, where $Q=1.293 \mathrm{MeV}$ is the neutron-proton mass difference. At $T \gg 1 \mathrm{MeV}$, $(n / p) \simeq 1$. As the temperature dropped, the neutronproton inter-conversion rate, $\Gamma_{n p} \sim G_{F}^{2} T^{5}$, fell faster than the Hubble expansion rate, $H \sim \sqrt{g_{*} G_{N}} T^{2}$. This resulted in breaking of chemical equilibrium ("freeze-out") at $T_{f r} \sim\left(g_{*} G_{N} / G_{F}^{4}\right)^{1 / 6} \simeq 0.8 \mathrm{MeV}$. The neutron fraction at this time, $n / p=e^{-Q / T_{f r}} \simeq 1 / 6$ is thus sensitive to every known physical interaction, since $Q$ is determined by both strong and electromagnetic interactions while $T_{f r}$ depends on the weak as well as gravitational interactions. Moreover, the sensitivity to the Hubble expansion rate affords a probe of e.g. the number of relativistic neutrino species [9]. After freeze-out the neutrons were free to $\beta$ decay so the neutron fraction dropped to $\simeq 1 / 7$ by the time nuclear reactions began. A useful semi-analytic description of freeze-out has been given in $[10,11]$.

The nucleosynthesis chain begins with the formation of deuterium in the process $p(n, \gamma) \mathrm{D}$. However, the number density of photons is huge relative to the baryon density, i.e., the baryon-to-photon ratio $\eta=n_{B} / n_{\gamma} \sim 10^{-9}$. Indeed, $\eta$ is the sole parameter in the standard BBN model.

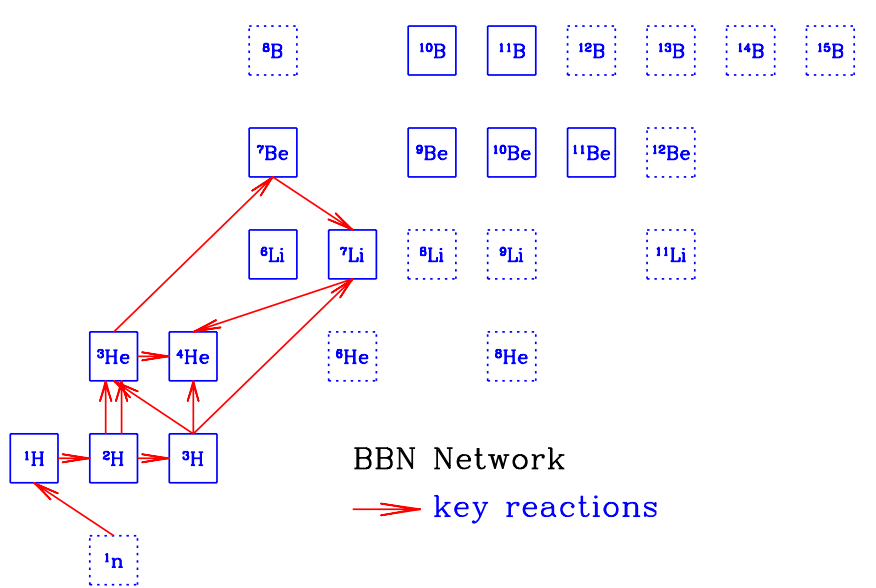

All reactions measured in lab at relevant energies

Fig. 1. An abbreviated nuclear network, showing the 12 most important reactions whose uncertainties dominate the theoretical error budget in BBN predictions. 


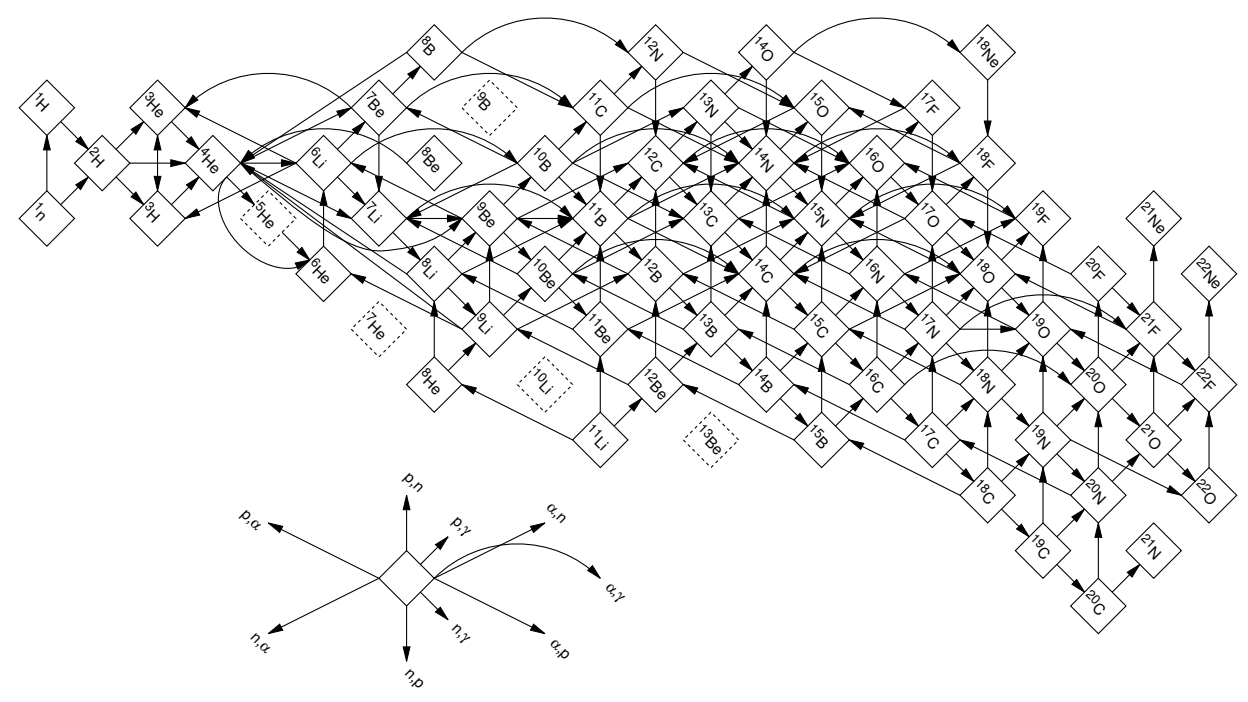

Fig. 2. The full nuclear network used in BBN calculations.

Because of the large number of photons per baryon, photodissociation delays production of deuterium (and other complex nuclei) well after $T$ drops below the binding energy of deuterium, $B_{\mathrm{D}}=2.23 \mathrm{MeV}$. The degree to which deuterium production is delayed can be found by comparing the qualitative expressions for the deuterium production and destruction rates,

$$
\begin{aligned}
& \Gamma_{p} \approx n_{B} \sigma v, \\
& \Gamma_{d} \approx n_{\gamma} \sigma v e^{-B_{\mathrm{D}} / T} .
\end{aligned}
$$

When the quantity $\eta^{-1} \exp \left(-B_{\mathrm{D}} / T\right) \sim 1$, the rate for deuterium destruction ( $\mathrm{D}+\gamma \rightarrow p+n)$ finally falls below the deuterium production rate and the nuclear chain begins at a temperature $T \sim 0.1 \mathrm{MeV}$. Only 2 -body reactions are important because the density has become rather low by this time.

In addition to the $p(n, \gamma) \mathrm{D}$ reaction, there are 10 other major strong reactions (along with the neutron lifetime) leading to the production of the light elements. These reactions, illustrated in fig. 1, are

$$
\begin{array}{ll}
\mathrm{D}(\mathrm{D}, p) \mathrm{T}, & { }^{3} \mathrm{He}(n, p) \mathrm{T}, \\
\mathrm{D}(\mathrm{D}, n)^{3} \mathrm{He}, & \mathrm{D}(p, \gamma)^{3} \mathrm{He} .
\end{array}
$$

Followed by the reactions producing ${ }^{4} \mathrm{He}$ :

$$
{ }^{3} \mathrm{He}(\mathrm{D}, p){ }^{4} \mathrm{He}, \quad \mathrm{T}(\mathrm{D}, n){ }^{4} \mathrm{He} .
$$

The gap at $A=5$ is overcome and the production and destruction of mass $A=7$ are regulated by

$$
\begin{aligned}
& { }^{3} \mathrm{He}\left({ }^{4} \mathrm{He}, \gamma\right){ }^{7} \mathrm{Be} \rightarrow{ }^{7} \mathrm{Li}+e^{+}+\nu_{e}, \\
& \mathrm{~T}\left({ }^{4} \mathrm{He}, \gamma\right){ }^{7} \mathrm{Li}, \quad{ }^{7} \mathrm{Be}(\mathrm{n}, \mathrm{p}){ }^{7} \mathrm{Li}, \quad{ }^{7} \mathrm{Li}\left(\mathrm{p},{ }^{4} \mathrm{He}\right){ }^{4} \mathrm{He} .
\end{aligned}
$$

The gap at $A=8$ prevents the production of other isotopes in any significant quantity. The nuclear chain in BBN calculations was extended [12] and is shown in fig. 2.
Nearly all the surviving neutrons when nucleosynthesis begins, end up bound in the most stable light element ${ }^{4} \mathrm{He}$. Heavier nuclei do not form in any significant quantity both because of the absence of stable nuclei with mass number 5 or 8 (which impedes nucleosynthesis via ${ }^{4} \mathrm{He}+n,{ }^{4} \mathrm{He}+p$ or ${ }^{4} \mathrm{He}+{ }^{4} \mathrm{He}$ reactions) and of the large Coulomb barriers for reactions such as the $T\left({ }^{4} \mathrm{He}, \gamma\right){ }^{7} \mathrm{Li}$ and ${ }^{3} \mathrm{He}\left({ }^{4} \mathrm{He}, \gamma\right){ }^{7} \mathrm{Be}$ reactions listed above. Hence the primordial mass fraction of ${ }^{4} \mathrm{He}$, conventionally referred to as $Y_{p}$, can be estimated by the simple counting argument

$$
Y_{p}=\frac{2(n / p)}{1+n / p} \simeq 0.25 .
$$

There is little sensitivity here to the actual nuclear reaction rates, which are however important in determining the other "left-over" abundances: D and ${ }^{3} \mathrm{He}$ at the level of a few times $10^{-5}$ by number relative to $\mathrm{H}$, and ${ }^{7} \mathrm{Li} / \mathrm{H}$ at the level of about $10^{-10}$ (when $\eta_{10} \equiv 10^{10} \eta$ is in the range 1-10). These values can be understood in terms of approximate analytic arguments $[13,11]$. The experimental parameter most important in determining $Y_{p}$ is the neutron lifetime, $\tau_{n}$, which normalizes (the inverse of) $\Gamma_{n p}$. (This is not fully determined by $G_{F}$ alone since neutrons and protons also have strong interactions, the effects of which cannot be calculated very precisely.) The experimental uncertainty in $\tau_{n}$ used to be a source of concern but has recently been reduced substantially. The Particle Data Group [14] world average is $\tau_{n}=885.7 \pm 0.8 \mathrm{~s}$.

Historically, BBN as a theory explaining the observed element abundances was nearly abandoned due to its inability to explain all element abundances. Subsequently, stellar nucleosynthesis became the leading theory for element production [15]. However, two key questions persisted. 1) The abundance of ${ }^{4} \mathrm{He}$ as a function of metallicity is nearly flat and no abundances are observed to be below about $23 \%$ as exaggerated in fig. 3 . In particular, even in systems in which an element such 


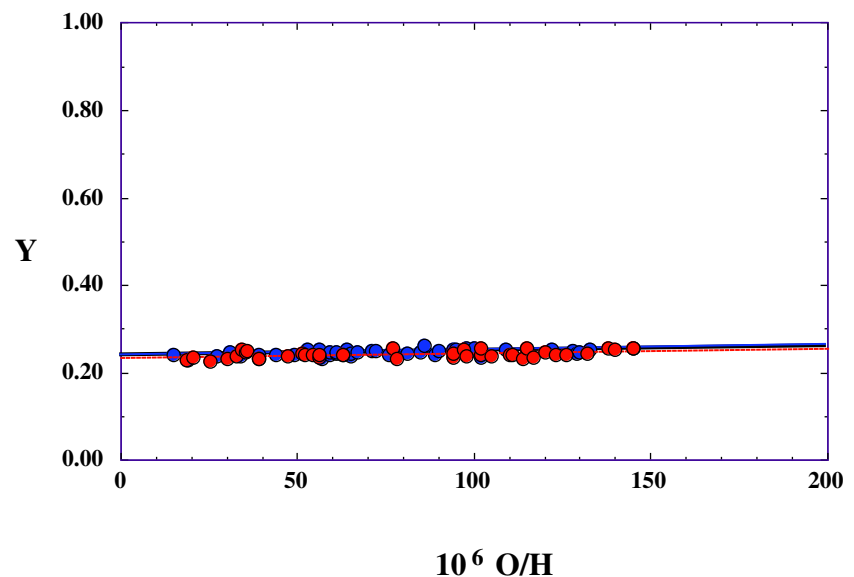

Fig. 3. The ${ }^{4} \mathrm{He}$ mass fraction as determined in extragalactic $\mathrm{HII}$ regions as a function of $\mathrm{O} / \mathrm{H}$.

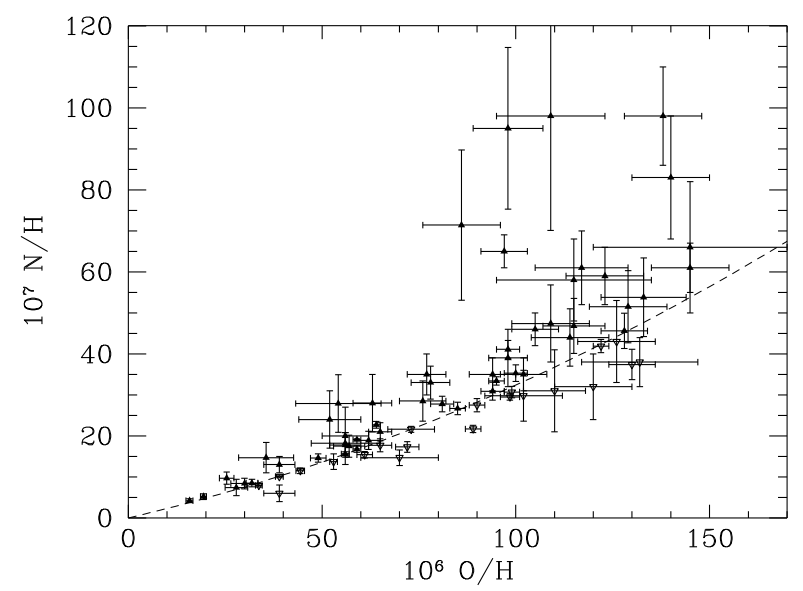

Fig. 4. The nitrogen and oxygen abundances in the same extragalactic HII regions with observed ${ }^{4}$ He shown in fig. 3.

as oxygen, which traces stellar activity, is observed at extremely low values (compared with the solar value of $\left.\mathrm{O} / \mathrm{H} \approx 8.5 \times 10^{-4}\right)$, the ${ }^{4} \mathrm{He}$ abundance is nearly constant. This is very different from all other element abundances (with the exception of ${ }^{7} \mathrm{Li}$ as we will see below). For example, in fig. 4, the $\mathrm{N} / \mathrm{H}$ vs. $\mathrm{O} / \mathrm{H}$ correlation is shown [16]. As one can clearly see, the abundance of $\mathrm{N} / \mathrm{H}$ goes to 0 , as $\mathrm{O} / \mathrm{H}$ goes to 0 , indicating a stellar source for nitrogen. 2) Stellar sources cannot produce the observed abundance of $\mathrm{D} / \mathrm{H}$. Indeed, stars destroy deuterium and no astrophysical site is known for the production of significant amounts of deuterium [17]. Thus we are led back to BBN for the origins of $\mathrm{D},{ }^{3} \mathrm{He},{ }^{4} \mathrm{He}$, and ${ }^{7} \mathrm{Li}$.

Having sketched the basic physics of BBN, we now turn to the detailed predictions and the nuclear data on which they rely.

\section{From nuclear data to primordial abundance predictions}

The homogeneous nature of BBN, and the relatively small number of key reactions, makes the abundance evolution one of the most computationally simple in all of nuclear astrophysics. This relative simplicity presents us with the opportunity to calculate the abundances with a precision and statistical rigor that also is unique to nuclear astrophysics. Given the increasingly precise CMB measurements, it is fortunate that BBN calculations (and to some extent light element observations) can keep pace.

Because standard BBN theory rests upon the Standard Model of particle physics, the electroweak aspects of the calculation are very well determined and do not introduce an appreciable uncertainty. Instead, the major uncertainties come from the thermonuclear reaction rates. As noted above, there are 11 key strong rates (as well as the neutron lifetime) which dominate the uncertainty budget $[18,19,20]$. In contrast to the situation for much of stellar nucleosynthesis, BBN occurs at high enough temperatures (strong rates freeze out at $T \sim 0.1 \mathrm{MeV}$ ) that laboratory data exist at and even below the relevant energies, so that no extrapolation is needed; this again places $\mathrm{BBN}$ in a unique position.

The goal of BBN theory is to derive primordial abundances which are as precise as the nuclear data allow, and to quantify the uncertainties in the predictions. This process takes several steps, going from the nuclear data to the final abundances. 1) First, the relevant nuclear data must be cataloged and evaluated. The data deemed reliable are used to infer both the best-fit cross section (in fact, the astrophysical $S$-factor), and to obtain an estimate of its uncertainty $\delta S$, both as functions of energy. 2) The cross section data are then averaged over a thermal Maxwell-Boltzmann distribution to obtain thermonuclear rates; similarly, one must propagate the cross section uncertainties to obtain thermonuclear errors. 3) Finally, the thermonuclear rates and uncertainties are placed in the BBN code. Monte Carlo techniques $[18,19]$ are used to determine the best-fit abundances, and their uncertainties, at each $\eta$.

Recently the input nuclear data have been carefully reassessed [20,21,22], leading to improved precision in the abundance predictions. In addition, polynomial fits to the predicted abundances and the error correlation matrix have been given [23,22].

A treatment of the nuclear and statistical aspects of the BBN calculation was presented by Cyburt [22]. The nuclear data was updated, and the cross sections and their uncertainties fit as a function of energy in a systematic and statistically consistent manner. The error propagation from energy-dependent cross sections to thermal rates was structured to explicitly include the effect of systematic errors in the different dataset normalizations, and to respond to statistically discrepant datasets. As emphasized in [20], systematic errors of these kinds dominate over the statistical errors in the nuclear data. Cyburt explicitly presents a set of thermonuclear rates and their temperature-dependent uncertainties, tailored for use in BBN calculations. He finds that because the systematic errors are indeed large, the results are quite similar to previous work [18] which have adopted the approximation in which these errors dominate. A reanalysis [24] in 

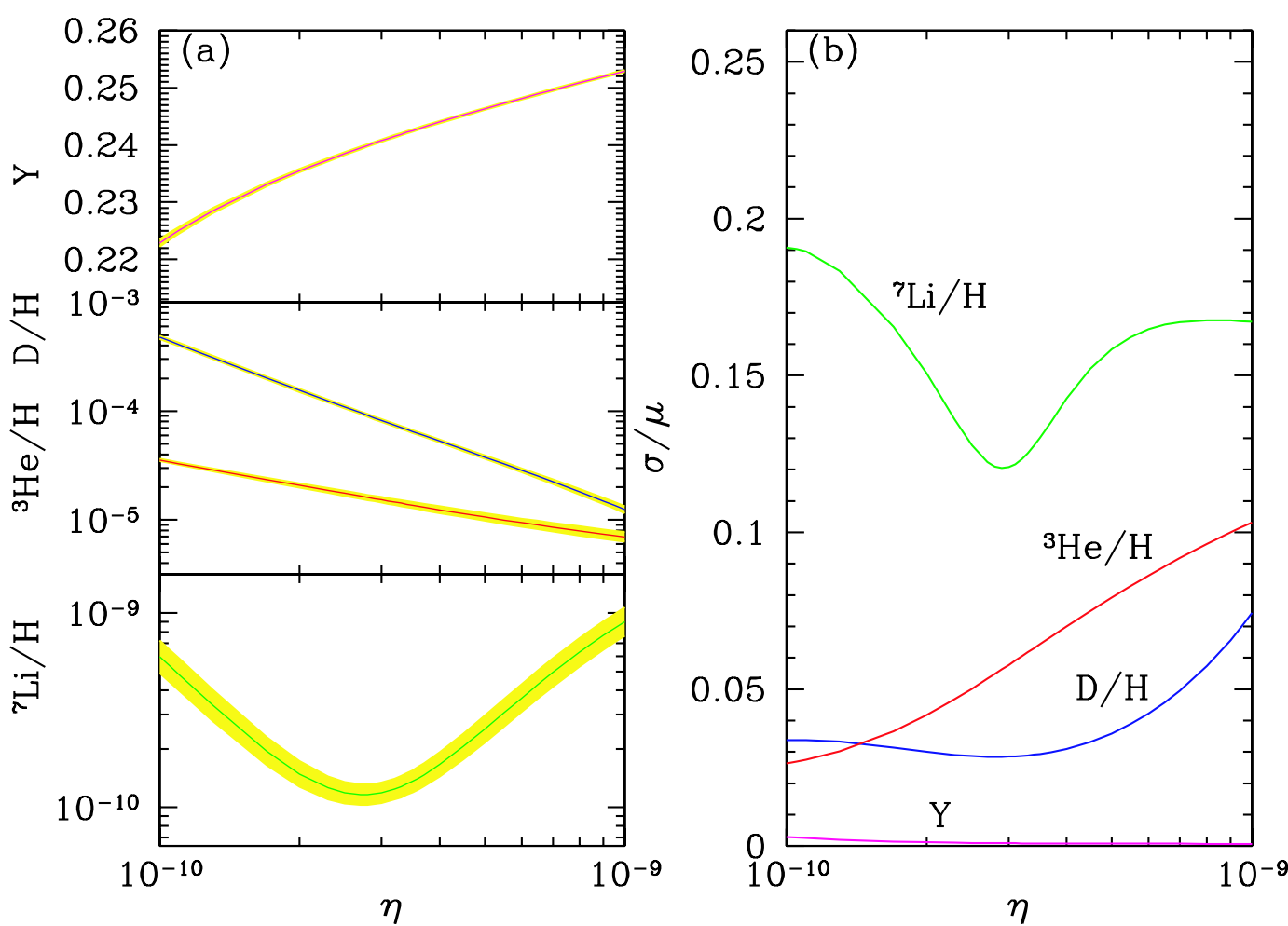

Fig. 5. The predictions of standard BBN [18], with thermonuclear rates based on the NACRE compilation [21]. (a) Primordial abundances as a function of the baryon-to-photon ratio $\eta$. Abundances are quantified as ratios to hydrogen, except for ${ }^{4} \mathrm{He}$ which is given in baryonic mass fraction $Y_{p}=\rho_{\mathrm{He}} / \rho_{B}$. The lines give the mean values, and the surrounding bands give the $1 \sigma$ uncertainties. (b) The $1 \sigma$ abundance uncertainties, expressed as a fraction of the mean value $\mu$ for each $\eta$.

the framework of $R$-matrix theory has recently been performed yielding comparable results.

The elemental abundances are shown in fig. 5 as a function of $\eta_{10}$ [18]. The left plot shows the abundance of ${ }^{4} \mathrm{He}$ by mass, $Y$, and the abundances of the other three isotopes by number. The curves indicate the central predictions from $\mathrm{BBN}$, while the bands correspond to the uncertainty in the predicted abundances. This theoretical uncertainty is shown explicitly in the right panel as a function of $\eta_{10}$. The uncertainty range in ${ }^{4} \mathrm{He}$ reflects primarily the $1 \sigma$ uncertainty in the neutron lifetime.

We first consider standard $\mathrm{BBN}$, which is based on Standard Model physics alone, so $N_{\nu}=3$. (The implications of BBN for physics beyond the Standard Model will be considered briefly below, sect. 5). As noted above, in the standard case, the only free parameter is the density of baryons (strictly speaking, nucleons), which sets the rates of the strong reactions. The baryon density is usually expressed normalized to the blackbody photon density as $\eta \equiv n_{B} / n_{\gamma}$; while both density change with time and temperature, their ratio $\eta$ remains constant from the end of BBN to the present. Because standard BBN is a one-parameter theory, any abundance measurement determines $\eta$, while additional measurements overconstrain the theory and thereby provide a consistency check. BBN has thus historically been the premier means of determining the cosmic baryon density.
Recently, however, a new "baryometer" has emerged in the form of the CMB. The release of the first-year WMAP results on the anisotropy spectrum of the CMB were a landmark event for all of cosmology, but particularly for $\mathrm{BBN}$. As with other cosmological parameter determinations from CMB data, the derived $\eta_{\mathrm{CMB}}$ depends on the adopted priors [25], in particular the form assumed for the power spectrum of primordial density fluctuations. If this is taken to be a scale-free power law, the WMAP data implies $\eta_{10}=6.58 \pm 0.27$, while allowing for a "running" spectral index lowers the value to

$$
\eta_{10}=6.14 \pm 0.25
$$

Equivalently, this can be stated as the allowed range for the baryon mass density today, $\rho_{B}=(4.2 \pm 0.2) \times$ $10^{-31} \mathrm{~g} \mathrm{~cm}^{-3}$, or as the baryonic fraction of the critical density: $\Omega_{B}=\rho_{B} / \rho_{\text {crit }} \simeq \eta_{10} h^{-2} / 274=(0.0224 \pm$ $0.0009) h^{-2}$, where $h \equiv H_{0} / 100 \mathrm{~km} \mathrm{~s}^{-1} \mathrm{Mpc}^{-1}$ is the present Hubble parameter.

The promise of CMB precision measurements of the baryon density suggests a new approach in which the CMB baryon density becomes an input to BBN. Thus, within the context of the Standard Model (i.e., with $N_{\nu}=3$ ), BBN becomes a zero-parameter theory, and the light element predictions are completely determined to within the uncertainties in $\eta_{\mathrm{CMB}}$ and the BBN theoretical errors. Comparison with light element observations then can be used to restate the test of $\mathrm{BBN}-\mathrm{CMB}$ consistency, or 
to turn the problem around and test the astrophysics of post-BBN light element evolution [26]. Alternatively, one can consider possible physics beyond the Standard Model (e.g., with $N_{\nu} \neq 3$ ) and then use all of the abundances to test such models; this is the subject of our final section.

\section{Light element observations and comparison with theory}

BBN theory predicts the universal abundances of $\mathrm{D},{ }^{3} \mathrm{He}$, ${ }^{4} \mathrm{He}$, and ${ }^{7} \mathrm{Li}$, which are essentially determined by $t \sim$ 180 s. Abundances are however observed at much later epochs, after stellar nucleosynthesis has commenced. The ejected remains of this stellar processing can alter the light element abundances from their primordial values, but also produce heavy elements such as $\mathrm{C}, \mathrm{N}, \mathrm{O}$, and Fe ("metals"). Thus one seeks astrophysical sites with low metal abundances, in order to measure light element abundances which are closer to primordial. For all of the light elements, systematic errors are an important and often dominant limitation to the precision of the primordial abundances.

In recent years, high-resolution spectra have revealed the presence of $\mathrm{D}$ in high-redshift, low-metallicity quasar absorption systems (QAS), via its isotope-shifted Lyman- $\alpha$ absorption. These are the first measurements of light element abundances at cosmological distances. It is believed that there are no astrophysical sources of deuterium [27], so any measurement of $\mathrm{D} / \mathrm{H}$ provides a lower limit to primordial $\mathrm{D} / \mathrm{H}$ and thus an upper limit on $\eta$; for example, the local interstellar value of $\mathrm{D} / \mathrm{H}=(1.5 \pm 0.1) \times 10^{-5}[28]$ requires that $\eta_{10} \leq 9$. In fact, local interstellar D may have been depleted by a factor of 2 or more due to stellar processing. However, for the high-redshift systems, conventional models of galactic nucleosynthesis (chemical evolution) do not predict significant $\mathrm{D} / \mathrm{H}$ depletion [29]; in this case, the high-redshift measurements recover the primordial deuterium abundance.

The five most precise observations of deuterium [30, $31,32,33]$ in QAS give $\mathrm{D} / \mathrm{H}=(2.78 \pm 0.29) \times 10^{-5}$, where the error is statistical only. These are shown in fig. 6 along with some other recent measurements $[34,35,36]$. Inspection of the data shown in the figure clearly indicates the need for concern over systematic errors. We thus conservatively bracket the observed values with a range $\mathrm{D} / \mathrm{H}=2-5 \times 10^{-5}$ which corresponds to a range in $\eta_{10}$ of 4-8 which easily brackets the CMB determined value. In principle, the steep decrease of $\mathrm{D} / \mathrm{H}$ with $\eta$ makes it a sensitive probe of the baryon density. We are optimistic that a larger sample of $\mathrm{D} / \mathrm{H}$ in high-redshift, low-redshift, and local systems will bring down systematic errors, and thereby increase the precision with which $\eta$ can be determined.

Using the WMAP value for the baryon density (5) the primordial $\mathrm{D} / \mathrm{H}$ abundance is predicted to be [7]:

$$
(\mathrm{D} / \mathrm{H})_{p}=2.75_{-0.19}^{+0.24} \times 10^{-5} \text {. }
$$

We note that the predicted value in eq. (6) is slightly higher than the value of $\mathrm{D} / \mathrm{H}=2.62_{-0.20}^{+0.18} \times 10^{-5}$ quoted

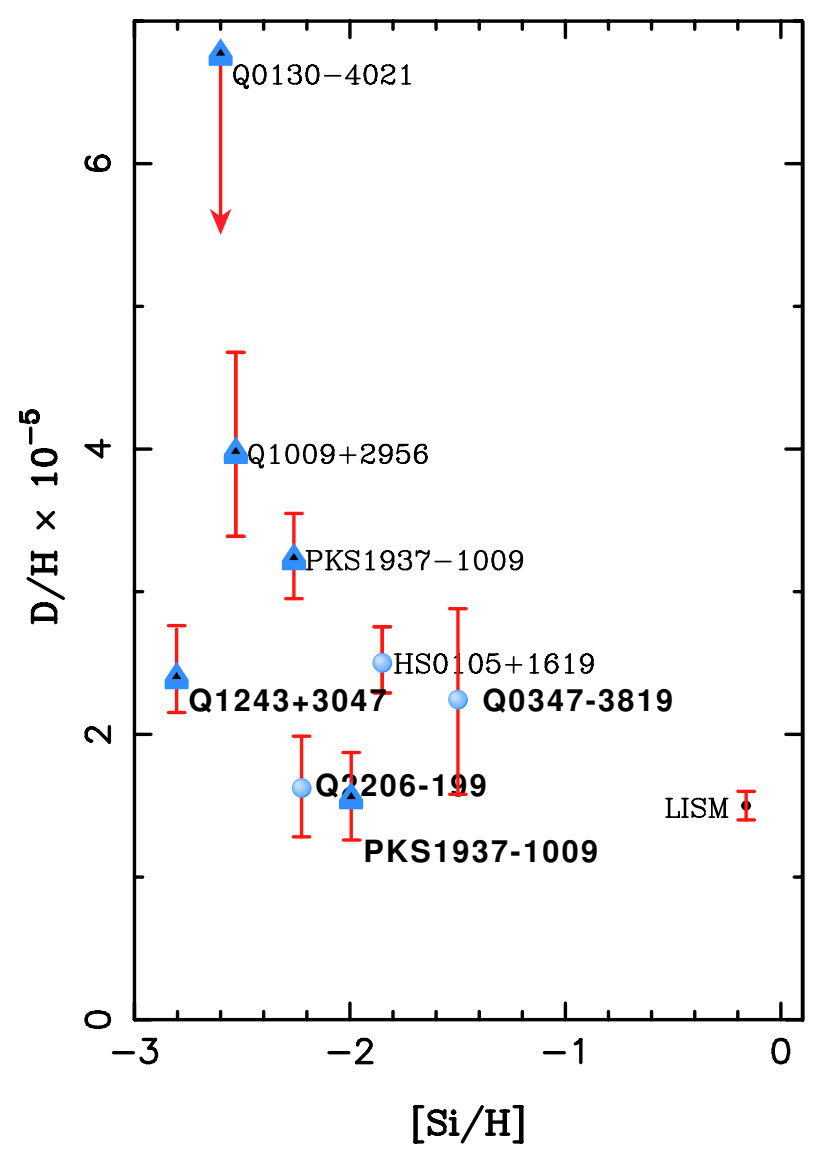

Fig. 6. $\mathrm{D} / \mathrm{H}$ abundances shown as a function of $[\mathrm{Si} / \mathrm{H}]$. Labels denote the background QSO, except for the local interstellar value (LISM; [28]).

in [6], this is largely due to our use of the most recent nuclear rates as determined by the most recent nuclear data compiled by the NACRE Collaboration [21]; at higher values of $\eta$, this leads to $5-10 \%$ more $\mathrm{D} / \mathrm{H}$ than older rates [26]. As one can see from fig. 7 (top left), this is in excellent agreement with the average of the 5 best determined quasar absorption system abundances noted above.

We observe ${ }^{4} \mathrm{He}$ in clouds of ionized hydrogen (HII regions), the most metal-poor of which are in dwarf galaxies. There is now a large body of data on ${ }^{4} \mathrm{He}$ and $\mathrm{CNO}$ in these systems [37]. These data confirm that the small stellar contribution to helium is positively correlated with metal production. ${ }^{4} \mathrm{He}$ abundance determinations depend on the overall intensity of the He emission line, but also on a number of physical parameters associated with the HII region. These include, the temperature, the electron density, optical depth and degree of underlying absorption. Previous extrapolations to zero metallicity have been based on various assumptions concerning these parameters and typically gave relatively low values for the primordial ${ }^{4} \mathrm{He}$ abundance. For example, a study using ${ }^{4} \mathrm{He}$ abundance where the electron density is derived from SII observations yielded $[16,38] Y_{p}=0.238 \pm 0.002 \pm 0.005$. Here the latter error is an estimate of the systematic uncertainty; this dominates, and is based on the scatter in 

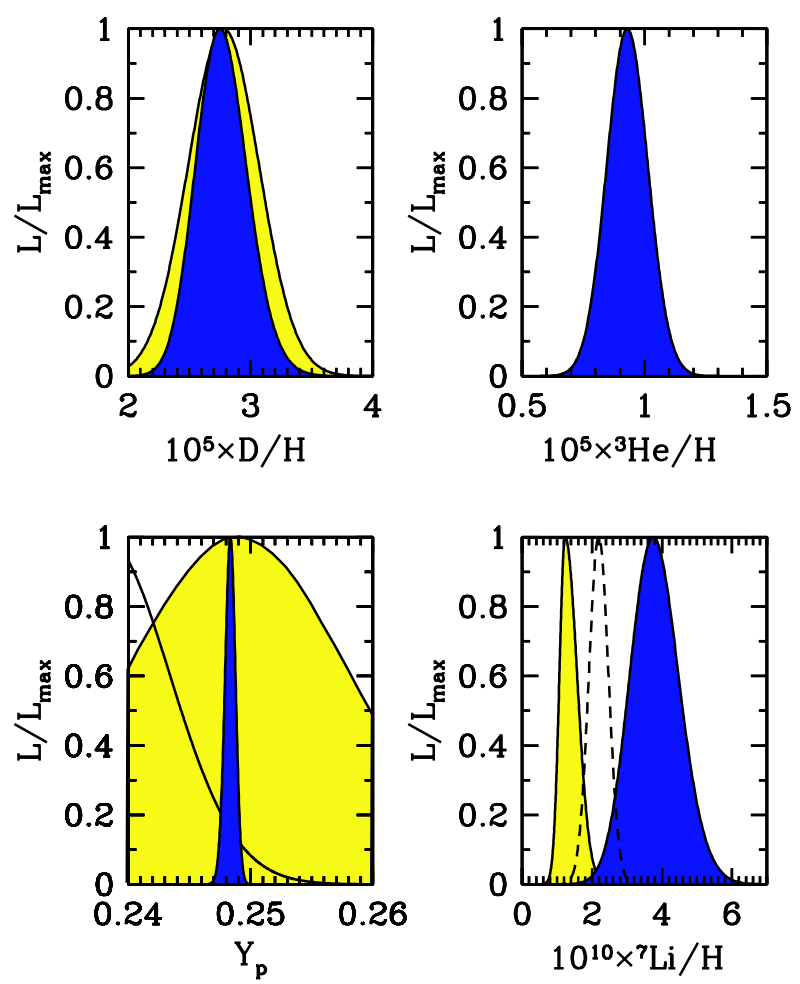

Fig. 7. Primordial light element abundances as predicted by BBN and WMAP (dark-shaded regions). Different observational assessments of primordial abundances are plotted as follows: (top left) the light-shaded region shows $\mathrm{D} / \mathrm{H}=$ $(2.78 \pm 0.29) \times 10^{-5}$; (top right) no observations plotted; (bottom left) the solid curve shows $Y_{p}=0.238 \pm 0.002 \pm 0.005$, and the light-shaded region shows $Y_{p}=0.249 \pm 0.009$; (bottom right) the light-shaded region shows ${ }^{7} \mathrm{Li} / \mathrm{H}=1.23_{-0.16}^{+0.34} \times 10^{-10}$, while the dashed curve shows ${ }^{7} \mathrm{Li} / \mathrm{H}=(2.19 \pm 0.28) \times 10^{-10}$.

different analyses of the physical properties of the HII regions $[37,39]$. Other extrapolations to zero metallicity give $Y_{p}=0.2443 \pm 0.0015$ [37], and $Y_{p}=0.2391 \pm 0.0020$ [40]. These are based on a self-consistent approach to determining the ${ }^{4} \mathrm{He}$ abundance. That is, several He lines are used to best fit the physical parameters. The effects of underlying absorption was not included in these analyses.

Recently a careful study of the systematic uncertainties in ${ }^{4} \mathrm{He}$, particularly the role of underlying absorption, has led to a higher value for the primordial abundance of ${ }^{4} \mathrm{He}$ [41]. Using a subset of the highest quality from the data of Izotov and Thuan [37], all of the physical parameters listed above including the ${ }^{4} \mathrm{He}$ abundance were determined self-consistently with Monte Carlo methods [39]. Note that the ${ }^{4} \mathrm{He}$ abundances are systematically higher, and the uncertainties are several times larger than quoted in [37]. In fact this study has shown that the value determined for $Y_{p}$ is highly sensitive to the method of analysis used. The extrapolated ${ }^{4} \mathrm{He}$ abundance was determined to be $Y_{p}=0.249 \pm 0.009$. Conservatively, it would be difficult at this time to exclude any value of $Y_{p}$ inside the range $0.232-0.258$.

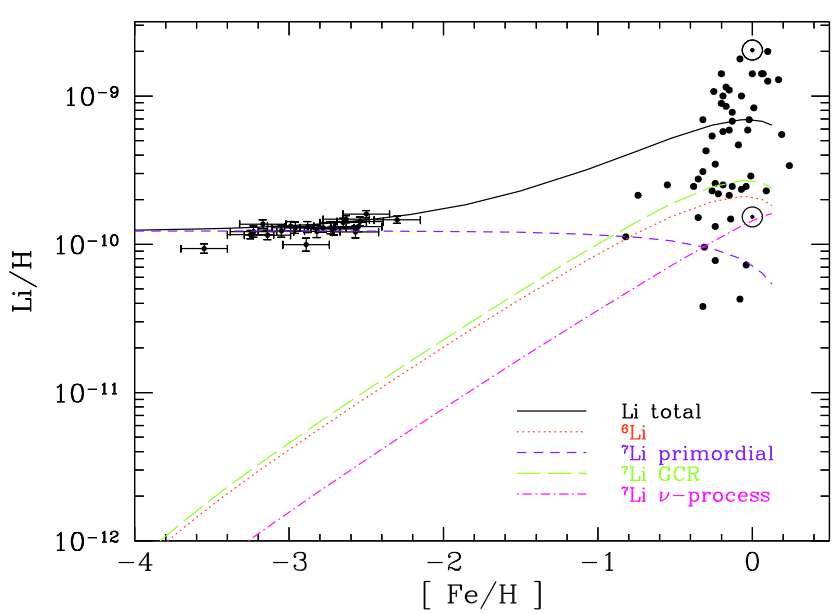

Fig. 8. Contributions to the total predicted lithium abundance from the adopted GCE model of [48], compared with low-metallicity stars and a sample of high-metallicity stars. The solid curve is the sum of all components.

At the WMAP value for $\eta$, the ${ }^{4} \mathrm{He}$ abundance is predicted to be [7]

$$
Y_{p}=0.2484_{-0.0005}^{+0.0004}
$$

While this value is considerably higher than any prior determination of the primordial ${ }^{4} \mathrm{He}$ abundance as can bee seen in fig. 7 (bottom left), it is in excellent agreement with the most recent analysis of the ${ }^{4} \mathrm{He}$ abundance [41]. Note also that the large uncertainty ascribed to this value indicates that while ${ }^{4} \mathrm{He}$ is certainly consistent with the WMAP determination of the baryon density, it does not provide for a highly discriminatory test of the theory at this time.

We also note that the CMB itself is sensitive to the helium abundance, which affects the mean number of electrons per baryon in the cosmic plasma. This opens the possibility that one can use the CMB to measure not only $\eta$ but also ${ }^{4} \mathrm{He}$, and thus test BBN entirely with the $\mathrm{CMB}$, without reference to low-redshift measurements and prior to any stellar nucleosynthetic contamination $[42,43]$. The issues of $Y_{p}$ degeneracy with other cosmological parameters remain to be sorted out, but we are optimistic that this approach may become an important new tool for BBN.

The systems best suited for Li observations are metalpoor stars in the spheroid (Pop II) of our Galaxy. Observations have long shown [44] that Li does not vary significantly in Pop II stars with metallicities $\lesssim 1 / 30$ of solar - the "Spite plateau". Recent precision data suggest a small but significant correlation between $\mathrm{Li}$ and Fe [45] which can be understood as the result of Li production from Galactic cosmic rays [46]. Extrapolating to zero metallicity one arrives at a primordial value [47] $\mathrm{Li} /\left.\mathrm{H}\right|_{p}=(1.23 \pm 0.06) \times 10^{-10}$.

Figure 8 shows the different $\mathrm{Li}$ components for a model with $\left({ }^{7} \mathrm{Li} / \mathrm{H}\right)_{p}=1.23 \times 10^{-10}$. The linear slope produced by the model is independent of the input primordial value. The model of ref. [48] includes in addition to primordial 
${ }^{7} \mathrm{Li}$, lithium produced in Galactic cosmic-ray nucleosynthesis (primarily $\alpha+\alpha$ fusion), and ${ }^{7} \mathrm{Li}$ produced by the $\nu$-process during type-II supernovae. As one can see, these processes are not sufficient to reproduce the population I abundance of ${ }^{7} \mathrm{Li}$, and additional production sources are needed.

The ${ }^{7} \mathrm{Li}$ abundance based on the WMAP baryon density is predicted to be [7]:

$$
{ }^{7} \mathrm{Li} / \mathrm{H}=3.82_{-0.60}^{+0.73} \times 10^{-10}
$$

or in astronomical notation, $A(\mathrm{Li}) \equiv \log _{10}(\mathrm{Li} / \mathrm{H})+12=$ $2.58_{-0.07}^{+0.08}$ dex. This value is in clear contradiction with most estimates of the primordial $\mathrm{Li}$ abundance as can be seen from fig. 7 (bottom right).

The quoted value for the ${ }^{7} \mathrm{Li}$ abundance assumes that the $\mathrm{Li}$ abundance in the stellar sample reflects the initial abundance at the birth of the star; however, an important source of systematic uncertainty comes from the possible depletion of Li over the $\gtrsim 10$ Gyr age of the Pop II stars. The atmospheric Li abundance will suffer depletion if the outer layers of the stars have been transported deep enough into the interior, and/or mixed with material from the hot interior; this may occur due to convection, rotational mixing, or diffusion. Standard stellar evolution models predict $\mathrm{Li}$ depletion factors which are very small $\left(\sigma_{A(\mathrm{Li})}<0.05 \mathrm{dex}\right)$ in very metal-poor turnoff stars [49]. However, there is no reason to believe that such simple models incorporate all effects which lead to depletion such as rotationally induced mixing and/or diffusion. Current estimates for possible depletion factors are in the range $\sim 0.2-0.4$ dex [50]. As noted above, this data sample [45] shows a negligible intrinsic spread in Li leading to the conclusion that depletion in these stars is as low as 0.1 dex.

Another important source for potential systematic uncertainty stems from the fact that the $\mathrm{Li}$ abundance is not directly observed but rather, inferred from an absorption line strength and a model stellar atmosphere. Its determination depends on a set of physical parameters and a model-dependent analysis of a stellar spectrum. Among these parameters, are the metallicity characterized by the iron abundance (though this is a small effect), the surface gravity which for hot stars can lead to an underestimate of up to $0.09 \mathrm{dex}$ if $\log g$ is overestimated by 0.5 , though this effect is negligible in cooler stars. Typical uncertainties in $\log g$ are $\pm 0.1-0.3$. The most important source for error is the surface temperature. Effective-temperature calibrations for stellar atmospheres can differ by up to $150-200 \mathrm{~K}$, with higher temperatures resulting in estimated $\mathrm{Li}$ abundances which are higher by $\sim 0.08$ dex per $100 \mathrm{~K}$. Thus accounting for a difference of 0.5 dex between $\mathrm{BBN}$ and the observations, would require a serious offset of the stellar parameters. We note however, that a recent study [51] with temperatures based on $\mathrm{H} \alpha$ lines (considered to give systematically high temperatures) yields ${ }^{7} \mathrm{Li} / \mathrm{H}=\left(2.19_{-0.38}^{+0.46}\right) \times 10^{-10}$ These results are based on a globular cluster sample and do show considerable dispersion. This result is consistent with previous Li measurements of the same cluster which gave ${ }^{7} \mathrm{Li} / \mathrm{H}=(1.91 \pm 0.44) \times 10^{-10}[52]$ and ${ }^{7} \mathrm{Li} / \mathrm{H}=$
$(1.69 \pm 0.27) \times 10^{-10}[53]$. A related study (also of globular cluster stars) gives ${ }^{7} \mathrm{Li} / \mathrm{H}=2.29 \times 10^{-10}$ [54].

Finally another potential source for systematic uncertainty lies in the BBN calculation of the ${ }^{7} \mathrm{Li}$ abundance. As one can see from fig. 5 , the predictions for ${ }^{7} \mathrm{Li}$ carry the largest uncertainty of the 4 light elements which stems from uncertainties in the nuclear rates. The effect of changing the yields of certain $\mathrm{BBN}$ reactions was recently considered by $\mathrm{Coc}$ et al. [55]. In particular, they concentrated on the set of cross sections which affect ${ }^{7} \mathrm{Li}$ and are poorly determined both experimentally and theoretically. In many cases however, the required change in cross section far exceeded any reasonable uncertainty. Nevertheless, it may be possible that certain cross sections have been poorly determined. In [55], it was found for example, that an increase of either the ${ }^{7} \mathrm{Li}(d, n) 2{ }^{4} \mathrm{He}$ or ${ }^{7} \mathrm{Be}(d, p) 2^{4} \mathrm{He}$ reactions by a factor of 100 would reduce the ${ }^{7} \mathrm{Li}$ abundance by a factor of about 3 .

The possibility of systematic errors in the ${ }^{3} \mathrm{He}(\alpha, \gamma){ }^{7} \mathrm{Be}$ reaction, which is the only important ${ }^{7} \mathrm{Li}$ production channel in BBN, was considered in detail in [56]. The absolute value of the cross section for this key reaction is known relatively poorly both experimentally and theoretically. However, the agreement between the standard solar model and solar neutrino data thus provides additional constraints on variations in this cross section. Using the standard solar model of Bahcall [57], and recent solar neutrino data [58], one can exclude systematic variations of the magnitude needed to resolve the $\mathrm{BBN}{ }^{7} \mathrm{Li}$ problem at the $\gtrsim 95 \%$ CL [56]. Thus the "nuclear fix" to the ${ }^{7} \mathrm{Li} \mathrm{BBN}$ problem is unlikely.

Finally, we turn to ${ }^{3} \mathrm{He}$. Here, the only observations available are in the solar system and (high-metallicity) HII regions in our Galaxy [59]. This makes inference of the primordial abundance difficult, a problem compounded by the fact that stellar nucleosynthesis models for ${ }^{3} \mathrm{He}$ are in conflict with observations [60]. Consequently, it is no longer appropriate to use ${ }^{3} \mathrm{He}$ as a cosmological probe [61]; instead, one might hope to turn the problem around and constrain stellar astrophysics using the predicted primordial ${ }^{3} \mathrm{He}$ abundance [62]. For completeness, we note that the ${ }^{3} \mathrm{He}$ abundance is predicted to be [7]:

$$
{ }^{3} \mathrm{He} / \mathrm{H}=9.28_{-0.54}^{+0.55} \times 10^{-6}
$$

at the WMAP value of $\eta$.

The overall concordance as evidenced in fig. 7 is encouraging and will be sharpened with further WMAP data, and subsequently from Planck Surveyor measurements. However, note that ${ }^{7} \mathrm{Li}$ is inconsistent with the $\mathrm{CMB}$ (as it is with $\mathrm{D}$ and ${ }^{4} \mathrm{He}$ ) given the error budgets we have quoted. The question then becomes more pressing as to whether this mismatch comes from systematic errors in the observed abundance predictions, or whether there might be new physics at work.

\section{Beyond the Standard Model}

Given the simple physics underlying BBN, it is remarkable that it still provides the most effective test for the 
cosmological viability of ideas concerning physics beyond the Standard Model. Limits on particle physics beyond the Standard Model come mainly from the observational bounds on the ${ }^{4} \mathrm{He}$ abundance. As discussed earlier, the neutron-to-proton ratio is fixed by its equilibrium value at the freeze-out of the weak interaction rates at a temperature $T_{f} \sim 1 \mathrm{MeV}$ modulo the occasional free neutron decay. Furthermore, freeze-out is determined by the competition between the weak interaction rates and the expansion rate of the universe

$$
G_{F}^{2} T_{f}^{5} \sim \Gamma_{\text {weak }}\left(T_{f}\right)=H\left(T_{f}\right) \sim \sqrt{G_{N} g_{*}} T_{f}^{2} .
$$

In the Standard Model, the number of relativistic particle species at $1 \mathrm{MeV}$ is $g_{*}=5.5+\frac{7}{4} N_{\nu}$, where 5.5 accounts for photons and $e^{ \pm}$, and $N_{\nu}$ is the number of (massless) neutrino flavors. The presence of additional neutrino flavors (or any other relativistic species) at the time of nucleosynthesis increases the overall energy density of the universe and hence the expansion rate leading to a larger value of $T_{f},(n / p)$, and ultimately $Y_{p}$. Because of the form of eq. (10) it is clear that just as one can place limits [9] on $N_{\nu}$, any changes in the weak or gravitational coupling constants can be similarly constrained (for a discussion see ref. [63]).

The helium curves in fig. 5 were computed taking $N_{\nu}=3$; the computed abundance scales as $\Delta Y_{\mathrm{BBN}} \simeq$ $0.013 \Delta N_{\nu}$ [10]. Clearly the central value for $N_{\nu}$ from BBN will depend on $\eta$, which is independently determined (with little sensitivity to $N_{\nu}$ ). Here we have taken the WMAP value for $\eta$. As one can see from fig. 7 (bottom left), if the best value for the observed primordial ${ }^{4} \mathrm{He}$ abundance is 0.249 , then, for $\eta_{10} \sim 6$, the central value for $N_{\nu}$ is very close to 3 . The dependence of the light element abundances on $N_{\nu}$ is shown in fig. 9 [26]. Because of the relatively large error on the newly determined value for $Y_{p}$, the $2 \sigma$ upper limit to $Y_{p}$ of 0.267 implies a conservative bound of

$$
N_{\nu} \lesssim 4.5
$$

Note that this bound is significantly weaker than previous bounds using smaller values for the primordial ${ }^{4} \mathrm{He}$ abundance.

It is also possible to use $\mathrm{D}$ to place an interesting limit on $N_{\nu}$ [7]. As seen in fig. 9, D is not as sensitive to $N_{\nu}$ as ${ }^{4} \mathrm{He}$ is, but nonetheless it does have a significant dependence. The relative error in the observed abundance of $\mathrm{D} / \mathrm{H}$ ranges from $7-10 \%$, depending on what systems are chosen for averaging. If the five most reliable systems are chosen, the peak of the $N_{\nu}$ likelihood distribution lies at $N_{\nu} \approx 3.0$, with a width of $\Delta N_{\nu} \approx 1.0$ as seen in fig. 10 . However, if we limit our sample to the two D systems that have had multiple absorption features observed, then the peak shifts to $N_{\nu} \approx 2.2$, with a width of $\Delta N_{\nu} \approx 0.7$ as shown by the dashed curve. Adopting the five-system D average, $\mathrm{D} / \mathrm{H}=(2.78 \pm 0.29) \times 10^{-5}$, we get the following constraints on $N_{\nu}$ :

$$
\begin{aligned}
\hat{N}_{\nu} & =3.02 \\
1.26<N_{\nu} & <5.22 \quad(95 \% \mathrm{CCL}) .
\end{aligned}
$$

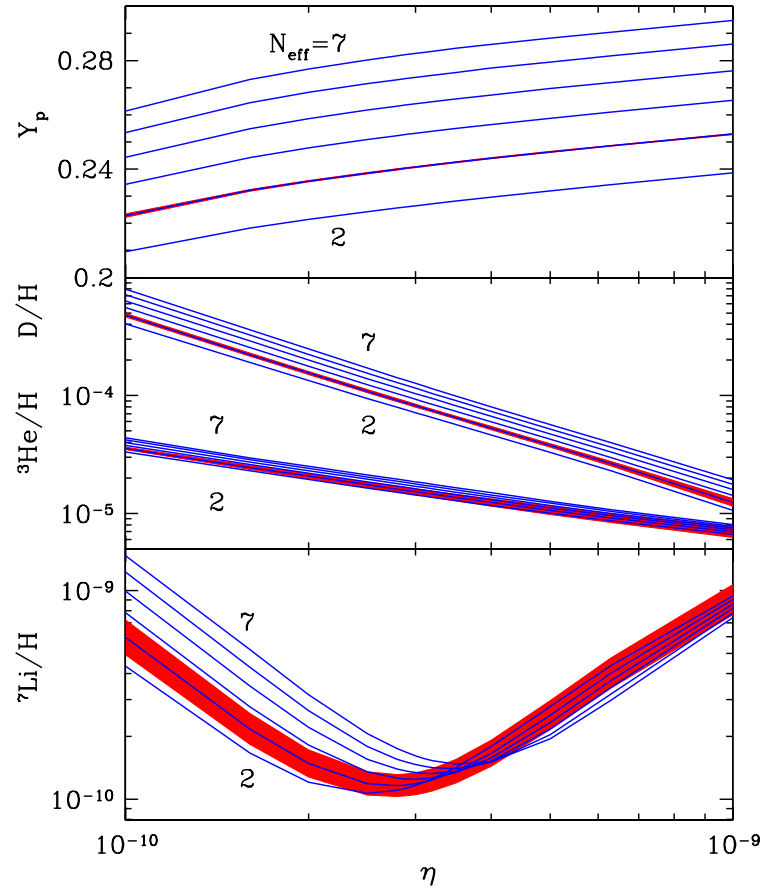

Fig. 9. BBN abundance predictions [26] as a function of the baryon-to-photon ratio $\eta$, for $N_{\nu}=2$ to 7 . The bands show the $1 \sigma$ error bars. Note that for the isotopes other than Li, the error bands are comparable in width to the thickness of the abundance curve shown. All bands are centered on $N_{\nu}=3$.

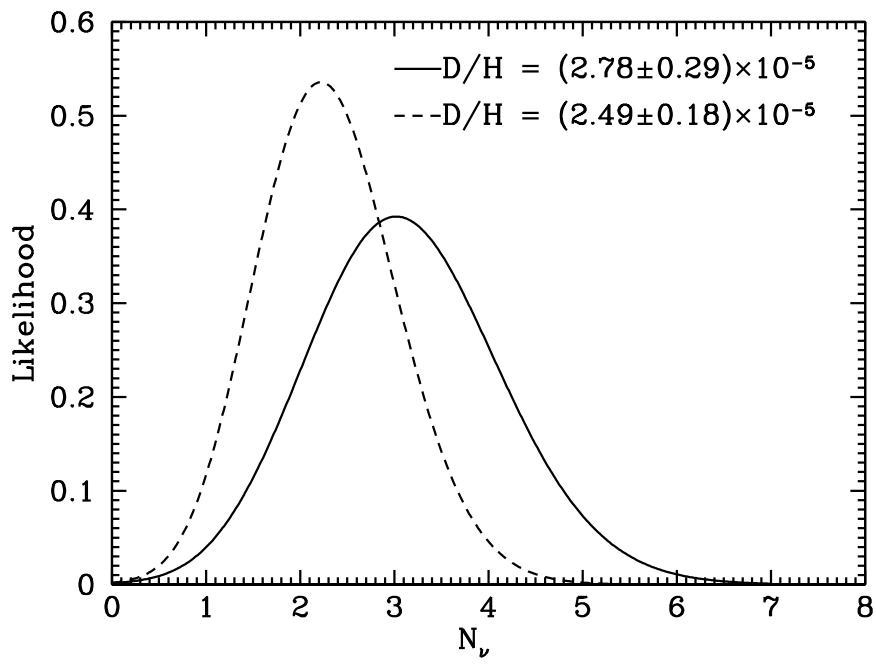

Fig. 10. Likelihoods for $N_{\nu}$ as predicted by the WMAP $\eta$ (eq. (5)) and light element observations as in fig. 7.

While the limit from $\mathrm{D} / \mathrm{H}$ remains weaker than the revised ${ }^{4} \mathrm{He}$ limit, it is certainly beginning to be competitive.

The limits on $N_{\nu}$ can be translated into limits on other types of particles or particle masses that would affect the expansion rate of the universe during nucleosynthesis. For example consider "sterile" neutrinos with only righthanded interactions of strength $G_{R}<G_{F}$. Such particles would decouple at higher temperature than (left-handed) 
neutrinos, so their number density $\left(\propto T^{3}\right)$ relative to neutrinos would be reduced by any subsequent entropy release, e.g. due to annihilations of massive particles that become non-relativistic in between the two decoupling temperatures. Thus (relativistic) particles with less than full strength weak interactions contribute less to the energy density than particles that remain in equilibrium up to the time of nucleosynthesis [64]. If we impose $N_{\nu}<4.5$ as an illustrative constraint, then the three right-handed neutrinos must have a temperature $3\left(T_{\nu_{R}} / T_{\nu_{L}}\right)^{4}<1.5$. The temperature of the decoupled $\nu_{R}$ 's is determined by entropy conservation, where $s=(4 / 3) \rho / T$ in a radiationdominated Universe. In analogy with the determination of the ratio of the left-handed neutrino temperature to the photon temperature $\left(T_{\nu_{L}} / T_{\gamma}=(4 / 11)^{1 / 3}\right.$, one finds $T_{\nu_{R}} / T_{\nu_{L}}=\left[(43 / 4) / g_{*}\left(T_{d}\right)\right]^{1 / 3}<0.84$, where $(43 / 4)$ is the value of $g_{*}$ at $T>1 \mathrm{MeV}$ including $\gamma, e^{ \pm}$, and $3 \nu_{L}$ 's and $T_{d}$ is the decoupling temperature of the $\nu_{R}$ 's. This requires $g_{*}\left(T_{d}\right)>18$. Since $g_{*}$ measures the number of relativistic species in thermal equilibrium, it increases with temperature as more particles are included in the radiation background. At the onset of BBN, $g_{*}=10.75$; to achieve $g_{*}>$ 18 requires the inclusion of quarks and gluons in the background, and thus one is forced to a temperature greater than the quark/hadron transition temperature or $T_{d}>$ $140 \mathrm{MeV}$. The decoupling temperature is related to $G_{R}$ through $\left(G_{R} / G_{F}\right)^{2} \sim\left(T_{d} / 3 \mathrm{MeV}\right)^{-3}$, where $3 \mathrm{MeV}$ is the decoupling temperature for $\nu_{L} \mathrm{~s}$. This yields a limit $G_{R} \lesssim$ $10^{-2} G_{F}$. This limit becomes sensitive to the assumed upper limit to $N_{\nu}$ when $T_{d}$ is constrained to be larger than the temperature of the quark-hadron transition.

\section{Summary}

As cosmology moves into a new and high-precision era, the utility of BBN is shifting from a test of the basic cosmological world model to one which is a sensitive probe of astrophysical processes which relate to the light element abundances as well as a sensitive probe of physics beyond the Standard Model. The parameters of the Standard Model as they relate to BBN are now well determined: $\eta_{10}=6.14$ and $N_{\nu}=3$. With these values, $\mathrm{BBN}$ is able to make very definite predictions for the primordial abundances of the light elements. These predictions are then contrasted with observational determinations of the abundances and reveal either our knowledge or ignorance of the astrophysical process which affect the primordial abundances. In the case of ${ }^{4} \mathrm{He}$ and $\mathrm{D} / \mathrm{H}$, within the large uncertainties in the observational data, the concordance is excellent. ${ }^{7} \mathrm{Li}$ remains problematic though several possible (if not perfect) astrophysical solutions are available. Finally, as the data on the light element abundances improves, BBN will sharpen its ability to probe physics beyond the Standard Model. As such it remains the only available tool for which to examine directly the very early universe.

The power and reach of BBN in the "new cosmology" rests upon the precision of its nuclear inputs, and of light element observations. Nuclear physicists can justly take pride that the cross sections used in BBN are now well measured, in the key cases to better than $10 \%$. However, as cosmology develops ever higher requirements for precision, BBN must also improve. Thus there is a need to improve all nuclear reaction data. More specifically, some reactions stand out as particularly in need of further examination. Notable examples are $n(p, \gamma) d$, which are well measured but not in the crucial BBN regime near $\sim 100 \mathrm{keV}$, and ${ }^{3} \mathrm{He}(\alpha, \gamma){ }^{7} \mathrm{Be}$ which dominates production of ${ }^{7} \mathrm{Li}$ and thus lies at the heart of the "lithium problem." Active work on these reactions was reported in NPA-II, and we look forward to seeing these efforts bear fruit by the NPA-III meeting.

I am grateful to the organizers for a stimulating and enjoyable meeting, and for being especially gracious hosts. It is a pleasure to thank my longtime BBN collaborators, particularly Keith Olive and Richard Cyburt. The work of B.D.F. was supported by the National Science Foundation under grant AST-0092939.

\section{References}

1. T.P. Walker, G. Steigman, D.N. Schramm, K.A. Olive, K. Kang, Astrophys. J. 376, 51 (1991); K.A. Olive, G. Steigman, T.P. Walker, Phys. Rep. 333, 389 (2000); B.D. Fields, S. Sarkar, Phys. Rev. D 66, 010001 (2002).

2. R.A. Malaney, G.J. Mathews, Phys. Rep. 229, 145 (1993).

3. S. Sarkar, Rep. Prog. Phys. 59, 1493 (1996).

4. G. Gamow, Nature 162, 680 (1948).

5. R.A. Alpher, R. Herman, Nature 162, 774 (1948).

6. C.L. Bennett et al., Astrophys. J. Suppl. 148, 1 (2003), arXiv:astro-ph/0302207; D.N. Spergel et al., Astrophys. J. Suppl. 148, 175 (2003), arXiv:astro-ph/0302209.

7. R.H. Cyburt, B.D. Fields, K.A. Olive, Phys. Lett. B 567, 227 (2003), arXiv:astro-ph/0302431.

8. B.D. Fields, K.A. Olive, to be published in Nucl. Phys. A.

9. G. Steigman, D.N. Schramm, J. Gunn, Phys. Lett. B 66, 202 (1977).

10. J. Bernstein, L.S. Brown, G. Feinberg, Rev. Mod. Phys. 61, 25 (1989)

11. V. Mukhanov, arXiv:astro-ph/0303073.

12. D. Thomas, D. Schramm, K.A. Olive, B. Fields, Astrophys. J. 406, 569 (1993).

13. R. Esmailzadeh, G.D. Starkman, S. Dimopoulos, Astrophys. J. 378, 504 (1991).

14. S. Eidelman et al. Phys. Lett. B 592, 1 (2004).

15. E.M. Burbidge, G.R. Burbidge, W.A. Fowler, F. Hoyle, Rev. Mod. Phys. 29, 547 (1957).

16. B.D. Fields, K.A. Olive, Astrophys. J. 506, 177 (1998).

17. H. Reeves, J. Audouze, W.A. Fowler, D.N. Schramm, Astrophys. J. 179, 909 (1973); R.I. Epstein, J.M. Lattimer, D.N. Schramm, Nature 263, 198 (1976); T. Prodanović, B.D. Fields, Astrophys. J. 597, 48 (2003), arXiv:astro$\mathrm{ph} / 0307183$.

18. R.H. Cyburt, B.D. Fields, K.A. Olive, New Astron. 6, 215 (2001), arXiv:astro-ph/0102179.

19. L.M. Krauss, P. Romanelli, Astrophys. J. 358, 47 (1990); M. Smith, L. Kawano, R.A. Malaney, Astrophys. J. Suppl. 85, 219 (1993); N. Hata, R.J. Scherrer, G. Steigman, D. Thomas, T.P. Walker, Astrophys. J. 458, 637 (1996); A. Coc, E. Vangioni-Flam, M. Casse, M. Rabiet, Phys. Rev. D 65, 043510 (2002). 
20. K.M. Nollett, S. Burles, Phys. Rev. D 61, 123505 (2000), arXiv:astro-ph/0001440.

21. NACRE Collaboration (C. Angulo et al.), Nucl. Phys. A 656, 3 (1999).

22. R.H. Cyburt, Phys. Rev. D 70, 023505 (2004), arXiv:astro$\mathrm{ph} / 0401091$.

23. G. Fiorentini, E. Lisi, S. Sarkar, F. L. Villante, Phys. Rev. D 58, 063506 (1998), arXiv:astro-ph/9803177; S. Burles, K.M. Nollett, M.S. Turner, Astrophys. J. 552, L1 (2001), arXiv:astro-ph/0010171.

24. P. Descouvemont, A. Adahchour, C. Angulo, A. Coc, E. Vangioni-Flam, arXiv:astro-ph/0407101.

25. M. Tegmark, M. Zaldarriaga, A.J.S. Hamilton, Phys. Rev. D 63, 043007 (2001) arXiv:astro-ph/0008167.

26. R.H. Cyburt, B.D. Fields, K.A. Olive, Astropart. Phys. 17, 87 (2002), arXiv:astro-ph/0105397.

27. R.I. Epstein, J.M. Lattimer, D.N. Schramm, Nature 263, 198 (1976).

28. J. Linsky, Space Sci. Rev. 84, 285 (1998).

29. D.D. Clayton, Astrophys. J. 290, 42 (1985); B.D. Fields, Astrophys. J. 456, 678 (1996).

30. S. Burles, D. Tytler, Astrophys. J. 499, 699 (1998); 507, $732(1998)$.

31. J.M. O'Meara, D. Tytler, D. Kirkman, N. Suzuki, J.X. Prochaska, D. Lubin, A.M. Wolfe, Astrophys. J. 552, 718 (2001), arXiv:astro-ph/0011179.

32. D. Kirkman, D. Tytler, N. Suzuki, J.M. O'Meara, D. Lubin, Astrophys. J. Suppl. 149, 1 (2003), arXiv:astro$\mathrm{ph} / 0302006$

33. M. Pettini, D.V. Bowen, Astrophys. J. 560, 41 (2001), arXiv:astro-ph/0104474.

34. D. Kirkman, D. Tytler, S. Burles, D. Lubin, J.M. O'Meara, Astrophys. J. 529, 655 (1999).

35. S. D'Odorico, M. Dessauges-Zavadsky, P. Molaro, Astron. Astrophys., 368, L21 (2001).

36. N.H. Creighton, J.K. Webb, A. Ortiz-Gill, A. FernandezSoto, astro-ph/0403512.

37. Y.I. Izotov, T.X. Thuan, V.A. Lipovetsky, Astrophys. J. 435, 647 (1994); Astrophys. J. Suppl. 108, 1 (1997); Y.I. Izotov, T.X. Thuan, Astrophys. J. 500, 188 (1998).

38. K.A. Olive, G. Steigman, Astrophys. J. Suppl. 97, 49 (1995), arXiv:astro-ph/9405022; K.A. Olive, E. Skillman, G. Steigman, Astrophys. J. 483, 788 (1997), arXiv:astro$\mathrm{ph} / 9611166$.

39. K.A. Olive, E.D. Skillman, New Astron. 6, 119 (2001).

40. M. Peimbert, A. Peimbert, M.T. Ruiz, Astrophys. J. 541, 688 (2000); A. Peimbert, M. Peimbert, V. Luridiana, Astrophys. J. 565, 668 (2002).

41. E.D. Skillman, K.A. Olive, Astrophys. J. 617, 29 (2004).

42. R. Trotta, S.H. Hansen, Phys. Rev. D 69, 023509 (2004), arXiv:astro-ph/0306588.
43. G. Huey, R.H. Cyburt, B.D. Wandelt, Phys. Rev. D 69 103503 (2004), arXiv:astro-ph/0307080.

44. F. Spite, M. Spite, Astron. Astrophys. 115, 357 (1982); P. Molaro, F. Primas, P. Bonifacio, Astron. Astrophys. 295, L47 (1995); P. Bonifacio, P. Molaro, Mon. Not. R. Astron. Soc. 285, 847 (1997).

45. S.G. Ryan, J.E. Norris, T.C. Beers, Astrophys. J. 523, 654 (1999).

46. B.D. Fields, K.A. Olive, New Astron., 4, 255 (1999); E. Vangioni-Flam, M. Cassé, R. Cayrel, J. Audouze, M. Spite, F. Spite, New Astron., 4, 245 (1999).

47. S.G. Ryan, T.C. Beers, K.A. Olive, B.D. Fields, J.E. Norris, Astrophys. J. Lett. 530, L57 (2000).

48. B.D. Fields, K.A. Olive, Astrophys. J. 516, 797 (1999).

49. C.P. Deliyannis, P. Demarque, S.D. Kawaler, Astrophys. J. Suppl. 73, 21 (1990).

50. S. Vauclair, C. Charbonnel, Astrophys. J. 502, 372 (1998); M.H. Pinsonneault, T.P. Walker, G. Steigman, V.K. Narayanan, Astrophys. J. 527, 180 (1998), arXiv:astro-ph/9803073; M.H. Pinsonneault, G. Steigman, T.P. Walker, V.K. Narayanan, Astrophys. J. 574, 398 (2002), arXiv:astro-ph/0105439

51. P. Bonifacio, et al., Astron. Astrophys. 390, 91 (2002).

52. L. Pasquini, P. Molaro, Astron. Astrophys. 307, 761 (1996).

53. F. Thevenin et al., Astron. Astrophys. 373, 905 (2001).

54. P. Bonifacio, Astron. Astrophys. 395, 515 (2002).

55. A. Coc, E. Vangioni-Flam, P. Descouvemont, A. Adahchour, C. Angulo, Astrophys. J. 600, 544 (2004), arXiv:astro-ph/0309480.

56. R.H. Cyburt, B.D. Fields, K.A. Olive, Phys. Rev. D 69 , 123519 (2004).

57. J.N. Bahcall, M.H. Pinsonneault, S. Basu, Astrophys. J. 555, 990 (2001).

58. SNO Collaboration (S.N. Ahmed et al.), Phys. Rev. Lett. 92, 181301 (2004), arXiv:nucl-ex/0309004.

59. D.S. Balser, T.M. Bania, R.T. Rood, T.L. Wilson, Astrophys. J. 510, 759 (1999).

60. K.A. Olive, D.N. Schramm, S.T. Scully, J.W. Truran, Astrophys. J. 479, 752 (1997), arXiv:astro-ph/9610039.

61. T.M. Bania, R.T. Rood, D.S. Balser, Nature 415, 54 (2002).

62. E. Vangioni-Flam, K.A. Olive, B.D. Fields, M. Casse, Astrophys. J. 585, 611 (2003), arXiv:astro-ph/0207583.

63. E.W. Kolb, M.J. Perry, T.P. Walker, Phys. Rev. D 33, 869 (1986); B.A. Campbell, K.A. Olive, Phys. Lett. B 345, 429 (1995); L. Bergstrom, S. Iguri, H. Rubinstein, Phys. Rev. D 60, 045005 (1999); C.M. Mueller, G. Schaefer, C. Wetterich, arXiv:astro-ph/0405373.

64. G. Steigman, K.A. Olive, D.N. Schramm, Phys. Rev. Lett. 43, 239 (1979); K.A. Olive, D.N. Schramm, G. Steigman, Nucl. Phys. B 180, 497 (1981). 